\title{
Analysis for forces in supports to exposed tendons in post-tensioned prestressed concrete bridges
}

\author{
C. B. WILBY
}

Major J. A. Jennings-Bramly. Civil Engineering Wing. Royal School of Military Engineering

The Paper gives a method which may be used to calculate support forces. However, the Author drops the $P e$ term between equations (1) and (2), thereby eventually deriving a deflexion equation independent of $P$ and $e$. If the $P e$ term is retained the deflexion equation for uniformly loaded and simply supported beam with tendons essentially parallel to the neutral axis may be simplified to

$$
y=\frac{\left(L_{1}^{2}-x^{2}\right)}{2 E I}\left[\frac{q}{12}\left(5 L_{1}^{2}-x^{2}\right)-P e\right](y \text { downwards }) \text {. . . . }
$$

14. The omission of live loads from the Author's analysis will often be correct because live load will merely reduce the hogging deformation due to prestress. However, in the Author's example a live load of, say, $9 \mathrm{kN} / \mathrm{m}$ changes the deflexion midspan to sagging, while nearer the span ends the curvature is still hogging (as one may expect from a quartic equation). The greatest tendon support forces are still reduced by this deformation but the support forces may also change direction due to the applied load. A greater live load will eventually increase the greatest tendon support force above that for the no live load case, but in the opposite direction.

15. It is possible that this method may be extended to include multispan beams and cable profiles bent at the tendon support points. The latter case will not often give relevant information.

\section{Professor Wilby}

I wrote the Paper relatively quickly. When the numerical part was solved and gave seemingly sensible practical answers, I assumed complete confidence in the theory. Usually numerical examples are very searching and expose any inadequacies in theories. In the present case, the slip in one term of the algebraic work did not appear to cause trouble in the numerical part. This one defect causes various differences in the Paper; $\$ \$ 2-10$ should be corrected to read as follows.

2. In Fig. 1 the tendons are supported at $\mathbf{A}, \mathbf{B}, \mathbf{C}$ and $\mathbf{D}$. Between these points the beam is slightly curved and the tendons droop slightly. As the supports are not very far apart and the force $P$ in the tendons is considerable, it is assumed 
that the eccentricity $e$ is constant. Then for any point co-ordinates $x, y$ on the line of the centroid of the beam

$$
M=R\left(L_{1}-x\right)-\frac{q}{2}\left(L_{1}-x\right)^{2}-P e=E I \frac{\mathrm{d}^{2} y}{\mathrm{~d} x^{2}} \quad \cdot \quad \cdot \quad .
$$

Thus

$$
\begin{gathered}
K_{1}+R L_{1} x-\frac{R x^{2}}{2}-\frac{q}{2} L_{1} x+\frac{q L_{1} x^{2}}{2}-\frac{q x^{3}}{6}-P e x=E I \frac{\mathrm{d} y}{\mathrm{~d} x} \\
K_{2}+K_{1} x+R L_{1} \frac{x^{2}}{2}-\frac{R x^{3}}{6}-\frac{q L_{1} x^{2}}{4}+\frac{q L_{1} x^{3}}{6}-\frac{q x^{4}}{24}-\frac{P e x^{2}}{2}=E I y
\end{gathered}
$$

3. When $x=0, \mathrm{~d} y / \mathrm{d} x=0$ and so from equation (2)

$$
K_{1}=0 \text {. }
$$

Resolving vertically for external forces

From equations (3)-(5)

$$
R=q L_{1}
$$

$$
K_{2}+\frac{q L_{1}^{2} x^{2}}{4}-\frac{q x^{4}}{24}-\frac{P e x^{3}}{2}=E I y \quad \text {. . . . . . . }
$$

When $x=L_{1}, y=0$ and so from equation (6)

$$
K_{2}=-\frac{5}{24} q L_{1}^{4}+\frac{P e L_{1}^{2}}{2}
$$

4. A prestressed concrete footbridge has the cross-section shown in Fig. 2. It is simply supported over a span of $30.48 \mathrm{~m}\left(=2 L_{1}\right)$ and is post-tensioned with the two groups of wires shown. These tendons are supported by five intermediate, equally spaced, supports attached to the vertical webs (or stems) of the girder. It is required to determine the force to be provided by the central supports, at transfer, for a total prestressing force after losses of $P=8000 \mathrm{kN}$, Young's modulus for concrete $E=34.5 \times 10^{8} \mathrm{kN} / \mathrm{m}^{2}$, and a weight density of concrete of $24 \mathrm{kN} / \mathrm{m}^{3}$.

5. The sectional properties are determined as in Table 1 (see reference 2).

6. The depth of the neutral axis below the top of the beam is

$$
\frac{0.753}{1.627}=0.4628 \mathrm{~m}
$$

The second moment of area about the neutral axis is

$$
0.776-1.627 \times 0.4628^{2}=I=0.4275 \mathrm{~m}^{4}
$$

The weight of the beam is

$$
1.627 \times 24=q=39.05 \mathrm{kN} / \mathrm{m}
$$

and the eccentricity of the prestressing force is

$$
\begin{gathered}
e=1.03-0.4628=0.5672 \mathrm{~m} \\
K_{2}=-\frac{5}{24} \times 39.05 \times\left(\frac{30.48}{2}\right)^{4}+\frac{8000 \times 0.5672}{2} \times\left(\frac{30.48}{2}\right)^{2}=88050
\end{gathered}
$$

7. From equation (6) the $y$ co-ordinate of $A^{\prime}$ in Fig. 1 is $(x=0)$

$$
\frac{K_{2}}{E I}=\frac{88050}{34.5 \times 10^{8} \times 0.4275}=5.970 \times 10^{-3}
$$

and that of $\mathrm{B}^{\prime}$ is $\left(x=L_{1} / 3\right)$

$$
\begin{aligned}
& \frac{1}{E I}\left[K_{3}+\frac{q L_{1}^{2}}{2}\left(\frac{L_{1}}{3}\right)^{2}-\frac{q L_{1}^{2}}{4}\left(\frac{L_{1}}{3}\right)^{2}-\frac{q}{24}\left(\frac{L_{1}}{3}\right)^{4}-\frac{P e}{2}\left(\frac{L_{1}}{3}\right)^{2}\right] \\
= & \frac{1}{E I}\left[K_{2}+0.02726 q L_{1}-\frac{P e}{2}\left(\frac{L_{1}}{3}\right)^{2}\right]=5.894 \times 10^{-3}
\end{aligned}
$$


8. The slope of the line $A B$ is the same as the slope of $A^{\prime} B^{\prime}$, i.e.

$$
10^{-3} \times(5 \cdot 894-5.970) /\left(\frac{L_{1}}{3}\right)=-0.00001496
$$

9. In Fig. $3 T$ is the force to be resisted by the two supports at $A$, and thus

$$
\tan \theta=0.00001496
$$

Therefore

$$
\sin \theta=0.00001496
$$

Resolving vertically

$$
T=2 P \sin \theta=2 \times 8000 \times 0.00001496=0.2394 \mathrm{kN}
$$

10. The forces to be resisted by supports at B, C and D can be calculated in a similar way.

17. I have been used to considering the theories propounded in reference 1 ; these concern the pressure between a tendon and its duct and this is greatest for design considerations when there is no live load and before grouting. This pressure is analogous to the forces in the tendon supports of the Paper. Likewise the greatest force in these supports was taken to be when no live load was present in the example, even though the tendons are not subsequently grouted in this case of exposed tendons. In this case of exposed tendons if the design were such that the deflexion was downwards at working loads then the forces in centrally disposed tendon supports would be upwards instead of downwards. I have used $q$ to represent total load, not necessarily just dead load. The theory applies to any loading. Most beams spend most of their lives without supporting full live load, so by the time they do, extra creep and shrinkage losses have occurred in the tendon force thus reducing the forces in the tendon supports. One naturally thinks of supporting the tendons to avoid drooping because the supports also have to carry the self-weight of the tendons. This weight adds to the maximum upwards force but subtracts from the maximum downwards force. Major JenningsBramly's point is a wise caution to the designer against just designing a support for maximum downwards force and not also for maximum upwards force. For example the designer will often not be right in just resting the tendons over a supporting bracket from the webs, but will need to tie them down to such a bracket.

18. The point about hogging near the supports while sagging at midspan is interesting. I thought about this mathematically by considering $\mathrm{d}^{2} y / \mathrm{d} x^{2}$, which is approximately the curvature. Equation (8) is the same as equations (6) and (7) except that the direction of $y$ is different. Differentiating this twice gives

$$
\frac{\mathrm{d}^{2} y}{\mathrm{~d} x^{2}}=\frac{1}{2 E I}\left(+q L_{1}^{2}-q x^{2}-2 P e\right) \text {. . . . . . . }
$$

19. If the curvature changes sign then the point of inflexion is when

$$
d^{2} y / d x^{2}=0
$$

j.e.

$$
+q L_{1}^{2}-q x^{2}-2 P e=0
$$

or

$$
2 P e / q=L^{2}-x^{2}
$$

20. For $x$ to be between $x=0$ and $x=L_{1}, L^{2}-x^{2}$ needs to be positive, so for any positive values of $P, e$ and $q$ (which would be a normal experience) the point of inflexion will be between $x=0$ and $x=L_{1}$.

21. For a hogging moment $\mathrm{d}^{2} y / \mathrm{d} x^{2}$ has to be negative, i.e.

$$
\left(L_{1}^{2}-x^{2}\right)-\frac{2 P e}{q}<0 \text {. . . . . . . . }
$$




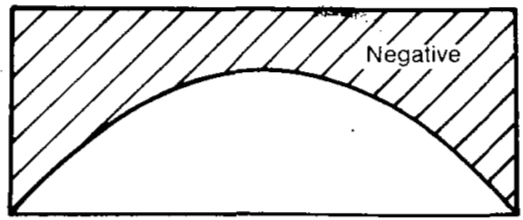

(a)

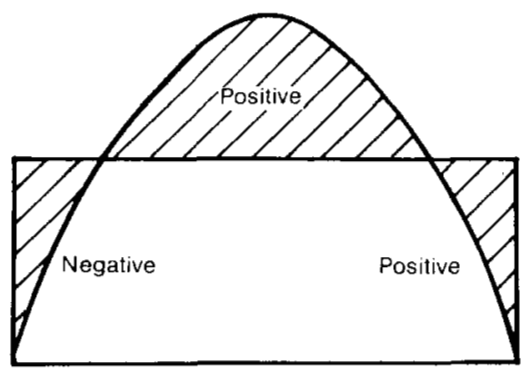

(b)

Fig. 4

For positive values of $P, e$ and $q$

(a) if $x^{2}=L_{1}^{2}-\Delta$, where $\Delta$ is a small quantity, equation (10) becomes $\Delta-2 P e / q<0$ which in practice occurs, so the curvature must be hogging

(b) if $x=0$ equation (10) becomes possible (i.e. hogging occurs) if $P e>q L_{1}{ }^{2} / 2$ and not possible (i.e. sagging occurs) if $P e<q L_{1}^{2} / 2$, as one would expect, as $P e$ and $q L_{1}{ }^{2} / 2$ are the midspan bending moments due to prestressing and vertical loading respectively.

22. A third option is to use an engineering approach rather than mathematics. The bending moment diagrams obtained by superimposing the bending moments due to prestressing and self-weight and total loading will generally be as shown in Figs 4(a) and 4(b) respectively. The beam in Fig. 4(a) will hog for the whole length of the span because the bending moment is always negative. The beam in Fig. 4(b) will sag for the central portion because of the positive bending moment and hog near the supports because of the negative bending moment.

23. The point which Major Jennings-Bramly raises thus applies to all simply supported prestressed concrete beams. It should be appreciated that all simply supported prestressed concrete beams hog near their supports even when fully loaded and overloaded. Does this mean that the deflexions at these locations are always upwards?

24. Using equations (6) and (7) and considering a point near a support, say $x^{2}=$ $L_{1}^{2}-\Delta$

$$
2 E I y=-\Delta\left[\frac{q}{12}\left(4 L_{1}^{2}+\Delta\right)-P e\right] \simeq-\Delta\left[\frac{q}{3} L_{1}^{2}-P e\right]
$$

Thus a point near the support deflects downwards if $q L_{1}{ }^{2} / 3$ is greater than $P e$ and upwards if $q L_{1}^{2} / 3$ is less than $P e$.

25. Alternatively from equations (6) and (7)

$$
\frac{\mathrm{d} y}{\mathrm{~d} x}=-\frac{1}{6 E I}\left[q x\left(x^{2}-3 L_{1}^{2}\right)+6 P e x\right] \text {. }
$$

26. For a point near a support, say $x=L_{1}-\Delta$

$$
6 E I \frac{\mathrm{d} y}{\mathrm{~d} x}=-\left\{q\left(L_{1}-\Delta\right)\left[\left(L_{1}-\Delta\right)^{2}-3 L_{1}^{2}\right]+6 P e\left(L_{1}-\Delta\right)\right\}
$$

Therefore

$$
3 E I \frac{\mathrm{d} y}{\mathrm{~d} x} \simeq q L_{1}{ }^{3}-3 P e L_{1}
$$


Thus a point near the support $\left(x=L_{1}\right)$ deflects downwards if $\mathrm{d} y / \mathrm{d} x$ is positive at $x=L_{1}$, i.e. if $q L_{1}{ }^{3}$ is greater than $3 P e L_{1}$ or $q L_{1}{ }^{2} / 3$ is greater than $P e$. Similarly a point near the support deflects upwards if $q L_{1}{ }^{2} / 3$ is less than $P e$.

27. In the beam of the example, $P e=8000 \times 0.5672=4538 \mathrm{kN} \mathrm{m}$ and $q L_{1}{ }^{2} / 3=$ $39.05 \times 15 \cdot 24^{2} / 3=3023 \mathrm{kN} \mathrm{m}$. Thus in this case the beam deflects upwards near the support.

28. In a beam designed according to $\mathrm{CP} 110^{3}$ in reference 2 , at working loads $P e=434.9 \mathrm{kN} \mathrm{m}$ and $q L_{1}{ }^{2} / 3=14.38 \times 11^{2} / 3=580.0 \mathrm{kN} \mathrm{m}$. Thus in this case the beam deflects downwards near its supports when loaded. At transfer $P e=503.7 \mathrm{kN}$ m and $q L_{1}^{2} / 3=6.018 \times 11^{2} / 3=242.7 \mathrm{kN} \mathrm{m}$, so the beam deflects upwards near its support.

29. The method can be used for continuous beams but it becomes more complicated and involves dealing with cable profiles deviated in construction at tendon support points.

\section{Roference}

3. Brrtish Standards Instrtution. The structural use of concrete. British Standards Institution, London, 1972, CP 110. 